\title{
REVIEW
}

\section{Function and Dysfunction of Adult Hippocampal Neurogenesis in Regeneration and Disease}

\author{
Lei Peng ${ }^{\star \dagger}$ and Michael A. Bonaguidi $* \$ \delta 币$
}

\begin{abstract}
From the Broad California Institute for Regenerative Medicine (CIRM) Center, ${ }^{*}$ the Department of Stem Cell Biology and Regenerative Medicine, ${ }^{\dagger}$ Neuroscience Graduate Program, and the Departments of Stem Cell Biology and Regenerative Medicine, ${ }^{\ddagger}$ Gerontology, ${ }^{\S}$ and Biomedical Engineering, ${ }^{\circledR}$ Zilkha Neurogenetic Institute, University of Southern California Keck School of Medicine, Los Angeles, California
\end{abstract}

\author{
Accepted for publication \\ September 21, 2017. \\ Address correspondence to Michael A. Bonaguidi, \\ $\mathrm{Ph} . \mathrm{D} .$, Broad California Institute for Regenerative \\ Medicine (CIRM) Center and Departments of \\ Stem Cell Biology and Regenerative Medicine, \\ Gerontology, and Biomedical Engineering, Zilkha \\ Neurogenetic Institute, University of Southern \\ California Keck School of Medicine, 1425 San \\ Pablo St, Broad CIRM Center 412, Los Angeles, \\ CA 90033. E-mail: mbonagui@usc.edu.
}

\begin{abstract}
The hippocampus is the only known brain region where physiological neurogenesis continues into adulthood across mammalian species and in humans. However, disease and injury can change the level of adult hippocampal neurogenesis, which plays an important role in regulating cognitive and emotional abilities. Alterations in hippocampal neurogenesis can mediate treatment of mental illness or affect the brain's capacity for repair and regeneration. In the present review, we evaluate how adult neurogenesis contributes to the repair and regeneration of hippocampal circuitry in the face of diseases and injuries. We also discuss possible future directions for harnessing adult neurogenesis for therapeutic use. (Am J Pathol 2018, 188: 23-28; https://doi.org/ 10.1016/j.ajpath.2017.09.004)
\end{abstract}

The adult brain has long been considered limited in its regenerative capacity in comparison with other organs or tissues in the body. For most of the 20th century, it was believed that neurogenesis ceased after development. However, in 1962, Altman ${ }^{1}$ injected adult rats with radioactive thymidine to label replicating cells and then demonstrated that mitotically active progenitors reside in the adult rat brain and give birth to new neurons. A series of subsequent studies over decades showed that adult neurogenesis exists in nearly all mammals, ${ }^{2}$ including humans. ${ }^{3,4}$ Under normal conditions, high levels of neurogenesis occur in two adult rodent regions: the subventricular zone of the lateral ventricles and the dentate gyrus of the hippocampus. Of the two regions, the dentate gyrus of the hippocampus is the only region capable of neurogenesis under basal conditions across mammalian species, including humans (Figure 1). This region's residing neural stem cells can generate functional new neurons and glia in response to pathologic and pharmacologic stimuli, maintaining both network plasticity and tissue homeostasis, with possible potential for repair and regeneration on disease and injury. 5,6

Adult hippocampal neurogenesis has been functionally linked to learning and memory and emotional processing, such as stress and depression. ${ }^{7-9}$ New neurons contribute to circuit remodeling in a 3- to 6-week critical period after their birth. ${ }^{5,6}$ Enhancing neurogenesis correlates with improved performance on learning and memory tests, such as the Morris and radialarm mazes, ${ }^{10,11}$ and during fear conditioning. ${ }^{12}$ On the other hand, ablated neurogenesis affects spatial and object recognition memory, fear conditioning, and pattern separation behaviors. ${ }^{13,14}$ In addition, hippocampal neurogenesis is highly regulated by the local and extrinsic environment. Stress suppresses neurogenesis through the corticosteroid and hypothalamic-adrenal axis. In turn, the level of neurogenesis also affects animals' sensitivity to stress. ${ }^{8,15}$ Moreover, physiological and pathologic factors (eg, age, exercise, environment, growth factors, hormones, injuries, and diseases) can influence the production and survival of new neurons in the dentate gyrus to alter hippocampal-dependent emotional and

Supported by NIH grant R00NS080913 and the L.K. Whittier Foundation (M.A.B.).

Disclosures: None declared.

This article is part of a review series on neural regeneration and developmental biology in health and disease. 
Sites of Adult Neurogenesis

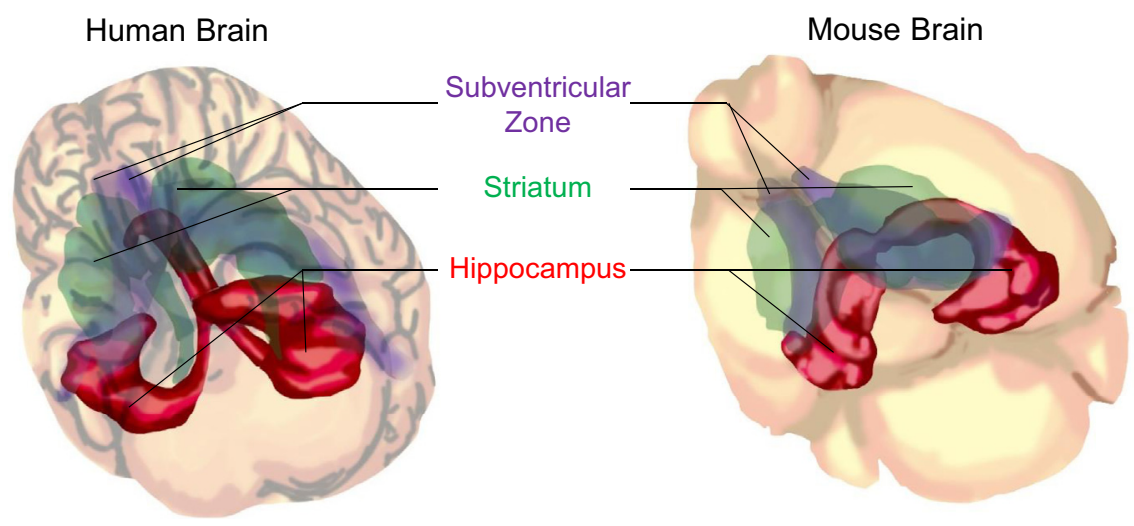

Figure 1 Adult neurogenesis regions. Adult neurogenesis is restricted in specific brain regions in humans and mice. Left panel: Human adult neurogenesis occurs under basal conditions in the hippocampus (red) and the striatum (green). Right panel: Murine adult neurogenesis occurs in the hippocampus (red) and the subventricular zone (purple). The hippocampus serves as the only brain region where adult neurogenesis is conserved across mammalian species. cognitive functions. ${ }^{16-18} \mathrm{We}$ will discuss both the positive and negative relationships among neurogenesis, postinjury repair, and neural disease development.

\section{Neurogenic Capacity Can Be Key for Positive Outcome}

The best described role for hippocampal neurogenesis in preventing disease occurs in mental health (Figure 2). The neurogenic hypothesis states that a lack of neurogenesis ${ }^{8}$ and synaptogenesis ${ }^{19}$ is a cause of depression. This premise is based on several observations: i) decreased hippocampal volume in patients and decreased neurogenesis in animal models of depression can be reversed by selective serotonin reuptake inhibitor antidepressants ${ }^{20}$; ii) depression-associated glucocorticoid elevation appears to inhibit neurogenesis ${ }^{21}$; and iii) selective serotonin reuptake inhibitor antidepressant effects have a delayed onset, consistent with the time frame required for newly generated neurons, despite rapid serotonin-level restoration. ${ }^{20}$ Most strikingly, studies ablating neurogenesis have shown selective serotonin reuptake inhibitors then lack an antidepressant effect. $^{22}$ Beyond mediating drug efficacy, ventral hippocampal lesions or neurogenic inhibition/depletion can produce animal models of psychiatric diseases, including depression, anxiety, and schizophrenia. Meanwhile, interventions, such as an enriched environment or exercise, can both restore neurogenesis and generate anxiolytic- and antidepressant-like effects. ${ }^{23}$ These studies strongly suggest that adult hippocampal neurogenesis serves as a key regulator for disease progression and can be used as a target for therapeutic drugs.

Neurogenesis also has importance for neural repair in the context of stroke. Disruption in the brain's blood supply because of hemorrhage or ischemia deprives glucose and oxygen and leads to an imbalance of prodeath and prosurvival signaling pathways. In the early stages after stroke, injury-induced endothelial or microglial cytokines trigger inflammation, which prolongs ischemic lesions. However, in later stages, the same endothelial and microglial cells produce beneficial factors, such as erythropoietin, to promote tissue repair. ${ }^{24}$ Patients experience transiently or permanently impaired facial recognition, calculation, or memory retrieval, and these disabilities are linked to cell death and loss of the ischemic and remote regions. ${ }^{25}$ Although early reperfusion is the main therapeutic target, thrombolysis is only suitable for $<5 \%$ of patients. On the other hand, spontaneous recovery is a common, yet inexplicable, phenomenon. In mice, rats, gerbils, monkeys, and humans with either focal or global ischemia, studies using a thymidine analog have shown hippocampal progenitor proliferation increases 48 hours after injury, peaks at 1 to 2 weeks, and continues until 3 to 4 weeks. ${ }^{26,27}$ The resulting newborn cells can migrate to the injury

$\begin{array}{ll} & \text { Hippocampal Neurogenesis } \\ \text { Beneficial } & \text { Detrimental }\end{array}$

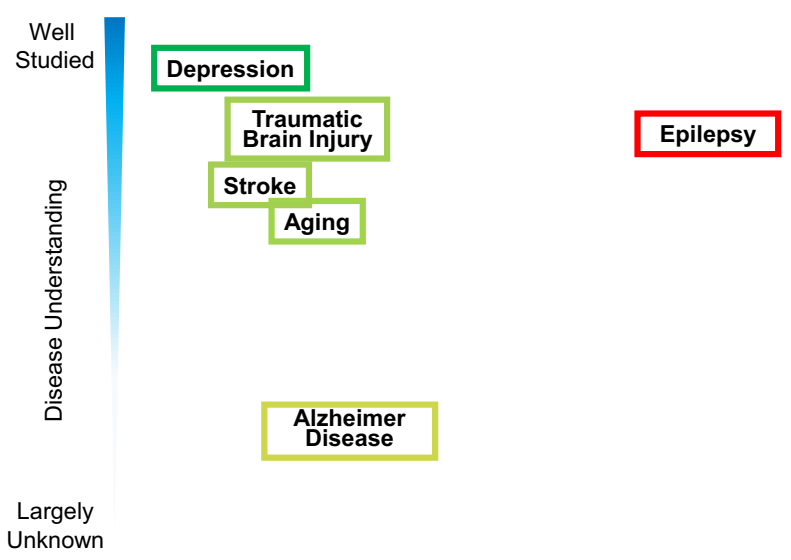

Figure 2 Roles of adult neurogenesis in disease. Hippocampal neurogenesis can be beneficial or detrimental to disease outcome, with varying degrees of data to support these interpretations. Depression is the most well-defined disease where adult neurogenesis acts as a beneficial contributor for treatment and symptom amelioration. Neurogenesis also contributes to more efficient repair and regeneration during stroke and traumatic brain injury. Meanwhile, neurogenesis in epilepsy can be detrimental to disease progression, and aging lowers the ability of neurogenesis to promote hippocampus repair. The conclusive establishment of neurogenesis in either disease progression or regeneration capacity remains largely unknown in Alzheimer disease and other neurodegenerative disorders. 
site and become differentiated neurons, expressing neuronal markers, such as doublecortin, polysialic acid neural cell adhesion molecule, neuronal nuclei, or calbindin. ${ }^{28}$ These newborn neurons are functionally important because inhibiting hippocampal neurogenesis in a gerbil model of bilateral common carotid artery occlusion yields impaired performance in water maze behavioral tests. ${ }^{29}$ The fact that the subgranular zone of the dentate gyrus serves as a steady reservoir of endogenous newborn neurons offers opportunities for therapeutic action. However, additional studies are needed to define whether surviving newborn neurons after stroke can integrate appropriately to replace the lost cells and, thus, improve functional recovery.

Many studies implicate neurogenic capacity in mediating partial recovery from traumatic brain injury (TBI). Head injuries can produce brain contusion, hemorrhage, and edema, leading to variable local or diffuse neuronal loss. ${ }^{30}$ Increased inflammation and reactive gliosis further impair remaining synaptic transmission and long-term potentiation to generate deficits in motor skills, attention, and memory. ${ }^{30}$ The hippocampus is the most frequently affected site that contributes to many of the cognitive deficits found in patients. ${ }^{31}$ Hippocampal neurogenesis is thought to partially mitigate this decline. Using rodent models, increases in proliferation and new neuron production occur as early as 2 days after injury. ${ }^{32}$ Injury-generated granular cells integrate into the existing hippocampal circuitry, ${ }^{33}$ and the level of endogenous neurogenesis correlates with the degree of innate cognitive recovery. Furthermore, administration of pharmacologic agents that increase neurogenesis after TBI improves functional outcome. ${ }^{34}$ These reports raise hope that TBI-induced neurogenesis may replace lost neurons, and enhancement to this process may heighten neurologic recovery. However, observed improved recovery may be caused by neurogenesis-independent neuroprotective effects. In addition, the potential repair process may be engaged under certain conditions and have long-term adverse consequences. For instance, increased neural stem cell proliferation is only observed in moderate or severe TBI. ${ }^{35}$ Neurogenesis has also been shown to peak at 1 to 2 weeks after injury and then subsides 2 to 12 weeks after injury. ${ }^{36}$ Injury-induced new neurons may also contain less complex morphology than under basal conditions and exhibit ectopic migration to the outer granule cell layer from 48 hours to 8 weeks after injury. ${ }^{37}$ These studies collectively indicate that injury-induced neurogenesis may have adverse long-term outcomes. Consistently, TBI increases the risk of epilepsy or neurodegenerative disease, ${ }^{30}$ indicating that neural damage still exists and/or that pathologically induced neurogenesis may undermine long-term neural function. Studies are needed to elucidate the function and underlying mechanisms of neural stem cells and these injury-induced newly generated neurons.

\section{Context Matters for Neurogenesis Consequence}

Increased neurogenesis is not always beneficial (Figure 2). Epilepsy is characterized by spontaneous recurrent motor seizures triggered by neuronal hyperactivity. At later disease stages, patients present many hippocampal functional deficits, including cell loss, cognitive impairment, depression, and higher rates of neurodegenerative diseases. It is well established that animal models of temporal lobe epilepsy exhibit dramatically elevated hippocampal neurogenesis because of enhanced progenitor proliferation and accelerated cell maturation, ${ }^{38}$ but they exhibit decreases in neurogenesis long-term. ${ }^{39}$ These findings also coincide with the observation of reduced cell proliferation in children with chronic seizures. ${ }^{40}$ Mechanistically, newly generated neurons migrate ectopically into the hilus or molecular cell layer, rather than to the granular cell layer, ${ }^{41}$ and produce abnormal dendritic synaptic contacts with GABAergic and granular cells instead of entorhinal cortex descending fibers. ${ }^{42}$ Furthermore, newborn neurons in the epileptic brain easily adapt to the hyperexcitable environment, and the remodeled hippocampal circuitry can worsen aberrant neuronal excitation. ${ }^{42}$ Neurogenesis removal by either applying antimitotic drugs or using transgenic models reduces long-term seizure frequency and cognitive impairment, providing additional support that abnormally integrated newly generated granule cells are proepileptogenic. $^{43}$

The timing of neurogenesis ablation and analysis can alter epileptic outcome. For example, Iyengar et $\mathrm{al}^{44}$ found that ablating neurogenesis conversely promoted acute vulnerability to epilepsy, increasing seizure frequency and duration. Moreover, neurogenesis ablation well after inducing epilepsy was not effective in reducing long-term seizures. ${ }^{43}$ Instead, specifically targeting newborn neurons a few days after inducing epilepsy decreases long-term seizure incidence but increases individual seizure duration. ${ }^{45}$ These differential outcomes reflect technical differences in modeling epilepsy and the cell types explored. Recently, Sierra et $\mathrm{al}^{46}$ varied dosages of the convulsant kainic acid in mice to model mild or strong seizure penetrance. They demonstrated that mild epilepsy chronically decreases neurogenesis, whereas strong forms additionally coax neural stem cells to generate reactive gliosis. As advanced temporal lobe epileptic patients often present hippocampal sclerosis, both newly generated neurons and astrocytes may differentially and collectively contribute to the interplay between new cell generation and epilepsy progression. This underlines the importance of understanding causes of epileptic pathology and designing therapeutic strategies according to the cellular context.

Age is another context that influences how adult neurogenesis contributes to repair and regeneration of hippocampal circuitry in the face of diseases and injuries. Because of better medical care and living conditions, increased life expectancy enhances the prevalence of aging as an identifiable risk factor for neural disease. Age-related neurogenesis decline coincides with an increasing incidence of neurodegeneration and a decreased regenerative capacity after injuries. Aging also decreases angiogenesis and blood flow, with accompanying brain activity declining in rats and monkeys. ${ }^{47}$ Interestingly, newborn neurons are still generated in old human brains, and the aged mouse brain retains 
some ability to regulate neurogenesis in response to macroenviromental changes, such as exercise. ${ }^{17}$ Furthermore, restoring trophic factors, including fibroblast growth factor 2 in the aged brain, ${ }^{48}$ decreasing humoral aging factors, including $\beta$-2-microglobulin, ${ }^{49}$ or injecting plasma from younger animals ${ }^{12}$ can promote neurogenesis and improve rodent cognitive function. Taken together, these findings imply that aged stem cells may still have the potential to aid in recovery from injury and disease but are restricted in how much they can grow and for how long. ${ }^{50}$ Identifying and releasing these mechanistic breaks can augment the application of adult neurogenesis for therapeutic use.

\section{Unclear Roles of Neurogenesis}

Adult hippocampal neurogenesis has long been suggested as a potential restorative mechanism to ameliorate cognitive decline induced by neural disorders. Neurodegenerative diseases exhibit cytotoxic protein aggregation and neuronal death, which can cause progressive deficits in behavioral and cognitive function. In Alzheimer disease (AD), the hippocampus is a principle affected brain region, contributing to cognitive decline and progressive dementia. ${ }^{51}$ Yet, transgenic AD models have produced conflicting reports about the role of endogenous neurogenesis: most studies implicate compromised neurogenesis, whereas others observe increased levels of new neuron generation. ${ }^{52}$ Akin to studies in epilepsy, these different observations may reflect when neurogenesis is analyzed. Another limitation is the choice of $\mathrm{AD}$ model. Most of the transgenic mice lack hippocampus neurodegeneration, with unclear and variable relationships between Alzheimer-like pathology and disease progression. ${ }^{53}$ However, manipulating the level of neurogenesis in $\mathrm{AD}$ models has effects on neuronal vulnerability and hippocampal-related cognitive function, supporting the hope that changes in neurogenesis may be restorative in $\mathrm{AD} .^{54}$ Other neurodegenerative diseases can have secondary effects on hippocampal neuronal loss, emotional or cognitive impairment, and neurogenesis regulation. In Parkinson disease, most pathologies are documented in the striatum and substantia nigra, but a reduction of progenitors and newborn neurons in the human hippocampus has also been described. ${ }^{55}$ Similarly, decreased hippocampal neurogenesis occurs in multiple rodent models of Huntington disease. ${ }^{56}$ These contexts indicate a possible link between neurodegeneration and adult neurogenesis, because both cytotoxic protein accumulation and deteriorating neurons may affect the birth of new neurons. ${ }^{57}$ Altogether, although neurogenesis is altered in a wide range of degenerative pathologies, limited evidence exists on whether it contributes to regeneration. ${ }^{58}$

\section{Perspective}

Endogenous neurogenesis promotes adaptability in response to environmental changes and potential to repair the neuronal network on injury or disease. In some contexts, such as depression, stroke, and TBI, our current understanding of hippocampal neurogenesis suggests feasibility for increasing recovery from these diseases. However, shortterm recovery mediated by neurogenesis may have longterm consequences, such as increased susceptibility to disease and neurodegeneration evidenced in TBI. Furthermore, neurogenesis itself may be co-opted by disease to advance pathology, as suggested in epilepsy. Moreover, epilepsy and aging are good examples that both the cellular context and influence of time matter to the design of using neurogenesis as a restorative therapy. Similarly, research on neurodegenerative diseases has produced inconclusive results about the role of hippocampal neurogenesis in $\mathrm{AD}$ and other disorders. Further efforts are warranted to elucidate microenvironmental mechanisms, their differential regulation of neurogenesis, and its relationship to disease. Moreover, greater knowledge is needed on the functional properties of insult-generated cells and their resultant behavior to promote positive outcome and recovery. Likewise, uncovering mechanisms that trigger or block cell proliferation and regulate differentiation or migration under pathologic conditions may propel the use of neural stem cells for effective therapies. By understanding the long-term consequences of neural stem cells' capacity to repair and regenerate, information about how to address acute pathologic need and how to maintain homeostasis and prolong healthy life may be gained. Such findings may help unleash an endogenous regenerative repertoire, with the least adverse effects in the hippocampus and other brain regions possessing neural disorder-induced neurogenesis. ${ }^{59}$

\section{Acknowledgments}

We thank Naibo Zhang for figure illustrations and Cristy Lytal for (Broad California Institute for Regenerative Medicine Center, University of Southern California, Los Angeles, CA). editing the manuscript.

\section{References}

1. Altman J: Are new neurons formed in the brains of adult mammals? Science 1962, 135:1127-1128

2. Rakic P, Nowakowski RS: The time of origin of neurons in the hippocampal region of the rhesus monkey. J Comp Neurol 1981, 196: 99-128

3. Eriksson PS, Perfilieva E, Bjork-Eriksson T, Alborn AM, Nordborg C, Peterson DA, Gage FH: Neurogenesis in the adult human hippocampus. Nat Med 1998, 4:1313-1317

4. Spalding KL, Bhardwaj RD, Buchholz BA, Druid H, Frisen J: Retrospective birth dating of cells in humans. Cell 2005, 122:133-143

5. Gage FH, Kempermann G, Palmer TD, Peterson DA, Ray J: Multipotent progenitor cells in the adult dentate gyrus. J Neurobiol 1998 , 36:249-266

6. van Praag $H$, Schinder AF, Christie BR, Toni N, Palmer TD, Gage FH: Functional neurogenesis in the adult hippocampus. Nature 2002, 415:1030-1034 
7. Squire LR, Ojemann JG, Miezin FM, Petersen SE, Videen TO, Raichle ME: Activation of the hippocampus in normal humans: a functional anatomical study of memory. Proc Natl Acad Sci U S A 1992, 89:1837-1841

8. Jacobs BL, van Praag H, Gage FH: Adult brain neurogenesis and psychiatry: a novel theory of depression. Mol Psychiatry 2000, 5:262-269

9. Revest JM, Dupret D, Koehl M, Funk-Reiter C, Grosjean N, Piazza PV, Abrous DN: Adult hippocampal neurogenesis is involved in anxiety-related behaviors. Mol Psychiatry 2009, 14:959-967

10. van Praag H, Christie BR, Sejnowski TJ, Gage FH: Running enhances neurogenesis, learning, and long-term potentiation in mice. Proc Natl Acad Sci U S A 1999, 96:13427-13431

11. Nilsson M, Perfilieva E, Johansson U, Orwar O, Eriksson PS: Enriched environment increases neurogenesis in the adult rat dentate gyrus and improves spatial memory. J Neurobiol 1999, 39:569-578

12. Villeda SA, Plambeck KE, Middeldorp J, Castellano JM, Mosher KI Luo J, Smith LK, Bieri G, Lin K, Berdnik D, Wabl R, Udeochu J, Wheatley EG, Zou B, Simmons DA, Xie XS, Longo FM, WyssCoray T: Young blood reverses age-related impairments in cognitive function and synaptic plasticity in mice. Nat Med 2014, 20:659-663

13. Clelland CD, Choi M, Romberg C, Clemenson GD, Fragniere A, Tyers P, Jessberger S, Saksida LM, Barker RA, Gage FH, Bussey TJ: A functional role for adult hippocampal neurogenesis in spatial pattern separation. Science 2009, 325:210-213

14. Saxe MD, Battaglia F, Wang JW, Malleret G, David DJ, Monckton JE, Garcia AD, Sofroniew MV, Kandel ER, Santarelli L, Hen R, Drew MR: Ablation of hippocampal neurogenesis impairs contextual fear conditioning and synaptic plasticity in the dentate gyrus. Proc Natl Acad Sci U S A 2006, 103:17501-17506

15. Snyder JS, Soumier A, Brewer M, Pickel J, Cameron HA: Adult hippocampal neurogenesis buffers stress responses and depressive behaviour. Nature 2011, 476:458-461

16. Kempermann G, Kuhn HG, Gage FH: More hippocampal neurons in adult mice living in an enriched environment. Nature 1997, 386: 493-495

17. van Praag H, Shubert T, Zhao C, Gage FH: Exercise enhances learning and hippocampal neurogenesis in aged mice. J Neurosci 2005, 25:8680-8685

18. Duman CH, Schlesinger L, Russell DS, Duman RS: Voluntary exercise produces antidepressant and anxiolytic behavioral effects in mice. Brain Res 2008, 1199:148-158

19. Duman RS, Li N: A neurotrophic hypothesis of depression: role of synaptogenesis in the actions of NMDA receptor antagonists. Philos Trans R Soc Lond B Biol Sci 2012, 367:2475-2484

20. Malberg JE, Eisch AJ, Nestler EJ, Duman RS: Chronic antidepressant treatment increases neurogenesis in adult rat hippocampus. J Neurosci 2000, 20:9104-9110

21. Gould E, Cameron HA, Daniels DC, Woolley CS, McEwen BS: Adrenal hormones suppress cell division in the adult rat dentate gyrus. J Neurosci 1992, 12:3642-3650

22. Santarelli L, Saxe M, Gross C, Surget A, Battaglia F, Dulawa S, Weisstaub N, Lee J, Duman R, Arancio O, Belzung C, Hen R: Requirement of hippocampal neurogenesis for the behavioral effects of antidepressants. Science 2003, 301:805-809

23. Duman RS, Malberg J, Nakagawa S, D'Sa C: Neuronal plasticity and survival in mood disorders. Biol Psychiatry 2000, 48:732-739

24. Marti HH, Bernaudin M, Petit E, Bauer C: Neuroprotection and angiogenesis: dual role of erythropoietin in brain ischemia. News Physiol Sci 2000, 15:225-229

25. Ardizzone TD, Zhan X, Ander BP, Sharp FR: SRC kinase inhibition improves acute outcomes after experimental intracerebral hemorrhage. Stroke 2007, 38:1621-1625

26. Liu J, Solway K, Messing RO, Sharp FR: Increased neurogenesis in the dentate gyrus after transient global ischemia in gerbils. J Neurosci 1998, 18:7768-7778

27. Jin K, Wang X, Xie L, Mao XO, Zhu W, Wang Y, Shen J, Mao Y, Banwait S, Greenberg DA: Evidence for stroke-induced neurogenesis in the human brain. Proc Natl Acad Sci U S A 2006, 103: 13198-13202

28. Tanaka R, Yamashiro K, Mochizuki H, Cho N, Onodera M, Mizuno Y, Urabe T: Neurogenesis after transient global ischemia in the adult hippocampus visualized by improved retroviral vector. Stroke 2004, 35:1454-1459

29. Raber J, Fan Y, Matsumori Y, Liu Z, Weinstein PR, Fike JR, Liu J: Irradiation attenuates neurogenesis and exacerbates ischemia-induced deficits. Ann Neurol 2004, 55:381-389

30. McKee AC, Daneshvar DH: The neuropathology of traumatic brain injury. Handb Clin Neurol 2015, 127:45-66

31. Girgis F, Pace J, Sweet J, Miller JP: Hippocampal neurophysiologic changes after mild traumatic brain injury and potential neuromodulation treatment approaches. Front Syst Neurosci 2016: 10:8

32. Chirumamilla S, Sun D, Bullock MR, Colello RJ: Traumatic brain injury induced cell proliferation in the adult mammalian central nervous system. J Neurotrauma 2002, 19:693-703

33. Emery DL, Fulp CT, Saatman KE, Schutz C, Neugebauer E, McIntosh TK: Newly born granule cells in the dentate gyrus rapidly extend axons into the hippocampal CA3 region following experimental brain injury. J Neurotrauma 2005, 22:978-988

34. Lu D, Mahmood A, Qu C, Goussev A, Schallert T, Chopp M: Erythropoietin enhances neurogenesis and restores spatial memory in rats after traumatic brain injury. J Neurotrauma 2005, 22:1011-1017

35. Wang X, Gao X, Michalski S, Zhao S, Chen J: Traumatic brain injury severity affects neurogenesis in adult mouse hippocampus. J Neurotrauma 2016, 33:721-733

36. Rice AC, Khaldi A, Harvey HB, Salman NJ, White F, Fillmore H, Bullock MR: Proliferation and neuronal differentiation of mitotically active cells following traumatic brain injury. Exp Neurol 2003, 183: 406-417

37. Kernie SG, Erwin TM, Parada LF: Brain remodeling due to neuronal and astrocytic proliferation after controlled cortical injury in mice. J Neurosci Res 2001, 66:317-326

38. Parent JM, Yu TW, Leibowitz RT, Geschwind DH, Sloviter RS, Lowenstein DH: Dentate granule cell neurogenesis is increased by seizures and contributes to aberrant network reorganization in the adult rat hippocampus. J Neurosci 1997, 17:3727-3738

39. Hattiangady B, Rao MS, Shetty AK: Chronic temporal lobe epilepsy is associated with severely declined dentate neurogenesis in the adult hippocampus. Neurobiol Dis 2004, 17:473-490

40. Mathern GW, Leiphart JL, De Vera A, Adelson PD, Seki T, Neder L, Leite JP: Seizures decrease postnatal neurogenesis and granule cell development in the human fascia dentata. Epilepsia 2002, 43 Suppl 5: $68-73$

41. Parent JM, von dem Bussche N, Lowenstein DH: Prolonged seizures recruit caudal subventricular zone glial progenitors into the injured hippocampus. Hippocampus 2006, 16:321-328

42. Koyama R, Tao K, Sasaki T, Ichikawa J, Miyamoto D, Muramatsu R, Matsuki N, Ikegaya Y: GABAergic excitation after febrile seizures induces ectopic granule cells and adult epilepsy. Nat Med 2012, 18: $1271-1278$

43. Cho KO, Lybrand ZR, Ito N, Brulet R, Tafacory F, Zhang L, Good L, Ure K, Kernie SG, Birnbaum SG, Scharfman HE, Eisch AJ, Hsieh J: Aberrant hippocampal neurogenesis contributes to epilepsy and associated cognitive decline. Nat Commun 2015, 6:6606

44. Iyengar SS, LaFrancois JJ, Friedman D, Drew LJ, Denny CA, Burghardt NS, Wu MV, Hsieh J, Hen R, Scharfman HE: Suppression of adult neurogenesis increases the acute effects of kainic acid. Exp Neurol 2015, 264:135-149

45. Hosford BE, Liska JP, Danzer SC: Ablation of newly generated hippocampal granule cells has disease-modifying effects in epilepsy. J Neurosci 2016, 36:11013-11023

46. Sierra A, Martin-Suarez S, Valcarcel-Martin R, Pascual-Brazo J, Aelvoet SA, Abiega O, Deudero JJ, Brewster AL, Bernales I, Anderson AE, Baekelandt V, Maletic-Savatic M, Encinas JM: 
Neuronal hyperactivity accelerates depletion of neural stem cells and impairs hippocampal neurogenesis. Cell Stem Cell 2015, 16:488-503

47. Small SA, Chawla MK, Buonocore M, Rapp PR, Barnes CA: Imaging correlates of brain function in monkeys and rats isolates a hippocampal subregion differentially vulnerable to aging. Proc Natl Acad Sci U S A 2004, 101:7181-7186

48. Rai KS, Hattiangady B, Shetty AK: Enhanced production and dendritic growth of new dentate granule cells in the middle-aged hippocampus following intracerebroventricular FGF-2 infusions. Eur $\mathrm{J}$ Neurosci 2007, 26:1765-1779

49. Smith LK, He Y, Park JS, Bieri G, Snethlage CE, Lin K, Gontier G, Wabl R, Plambeck KE, Udeochu J, Wheatley EG, Bouchard J, Eggel A, Narasimha R, Grant JL, Luo J, Wyss-Coray T, Villeda SA: beta2-Microglobulin is a systemic pro-aging factor that impairs cognitive function and neurogenesis. Nat Med 2015, 21:932-937

50. Roh JK, Jung KH, Chu K: Adult stem cell transplantation in stroke: its limitations and prospects. Curr Stem Cell Res Ther 2008, 3: 185-196

51. Braak H, Braak E: Neuropathological stageing of Alzheimer-related changes. Acta Neuropathol 1991, 82:239-259

52. Mu Y, Gage FH: Adult hippocampal neurogenesis and its role in Alzheimer's disease. Mol Neurodegener 2011, 6:85
53. Cuadrado-Tejedor M, Garcia-Osta A: Current animal models of Alzheimer's disease: challenges in translational research. Front Neurol 2014, 5:182

54. Jin K, Galvan V, Xie L, Mao XO, Gorostiza OF, Bredesen DE, Greenberg DA: Enhanced neurogenesis in Alzheimer's disease transgenic (PDGF-APP(Sw,Ind)) mice. Proc Natl Acad Sci U S A 2004, 101:13363-13367

55. Hoglinger GU, Rizk P, Muriel MP, Duyckaerts C, Oertel WH, Caille I, Hirsch EC: Dopamine depletion impairs precursor cell proliferation in Parkinson disease. Nat Neurosci 2004, 7:726-735

56. Gil JM, Mohapel P, Araujo IM, Popovic N, Li JY, Brundin P, Petersen A: Reduced hippocampal neurogenesis in R6/2 transgenic Huntington's disease mice. Neurobiol Dis 2005, 20:744-751

57. Steiner B, Wolf S, Kempermann G: Adult neurogenesis and neurodegenerative disease. Regen Med 2006, 1:15-28

58. Dietrich J, Kempermann G: Role of endogenous neural stem cells in neurological disease and brain repair. Adv Exp Med Biol 2006, 557: $191-220$

59. Krook-Magnuson E, Szabo GG, Armstrong C, Oijala M, Soltesz I: Cerebellar directed optogenetic intervention inhibits spontaneous hippocampal seizures in a mouse model of temporal lobe epilepsy. eNeuro 2014, 1. pii: e.2014 